\title{
REVIEW \\ A systematic review of risk factors for the development and recurrence of pressure ulcers in people with spinal cord injuries
}

\author{
$\mathrm{J} \mathrm{Marin}^{1}, \mathrm{~J}$ Nixon ${ }^{2}$ and C Gorecki ${ }^{2}$
}

Study design: A systematic review was undertaken, based upon methods recommended for effectiveness questions but adapted to identify observational risk factor (RF) studies.

Objectives: The literature identifies many RFs for pressure ulcer (PU) recurrence and development; however, RFs independently predictive of PU development in adults with spinal cord injury (SCI) have not been determined. A systematic review was undertaken to identify RFs for PUs for people with SCl.

Setting: Acute hospital, community and rehabilitation settings.

Methods: Electronic searches of MEDLINE, EMbase and Cochrane databases from 1980 to 2011 were completed. Retrieved studies were assessed for eligibility and quality criteria applied by two independent reviewers. Identified RFs were categorised into themes and compared and contrasted with RFs identified for the general PU population.

Results: The five studies included 18 RFs. These were classified into six themes: sociodemographic, neurological, functional, clinical, biological and medical care management. RFs for both the general and SCl-specific populations were similar, however, clinical, functional and hospital management emerged as specific RF domains for the SCl population.

Conclusion: We identified SCl-specific RFs for the development and recurrence of PUs. However, these findings are based on a small number of studies; highlighting the need for further confirmatory work to reduce PU development and recurrence, and provide a foundation for $\mathrm{SCl}$ risk assessment development.

Spinal Cord (2013) 51, 522-527; doi:10.1038/sc.2013.29; published online 16 April 2013

Keywords: spinal cord injury; pressure ulcer; risk factor; systematic review

\section{INTRODUCTION}

A pressure ulcer $(\mathrm{PU})$ is an area of localised damage to the skin as a result of prolonged pressure alone or pressure in combination with shearing forces. ${ }^{1}$ PUs vary in size and severity of tissue layer affected and range from skin erythema to damage to muscle and underlying bone. ${ }^{2}$ PUs are prevalent in all health-care settings. In Europe, USA and Canada, incidence rates range from 1 to $66 \%$ for hospital, 4.2 to $23.9 \%$ for nursing home and 1.5 to $25 \%$ for community hospital patients. $^{3-6}$ The cost of PUs to the National Health Service is substantial, with the total annual cost for treatment in the United Kingdom being £1.4-£2.1 billion, making up $4 \%$ of the annual National Health Service budget. ${ }^{7}$ This equates to approximately 412000 individuals in the United Kingdom developing PUs per annum. Clinical practice guidelines aim to minimise incidence by highlighting patients at risk and implementing patient-orientated interventions to prevent $\mathrm{PU}$ development, ${ }^{8}$ thereby reducing the burden of PUs on both patients and health-care systems.

The loss of motor, sensory and autonomic control associated with a spinal cord injury (SCI) makes PUs the most common secondary medical complication associated with such an injury; $;{ }^{910}$ up to $95 \%$ of adults with a SCI will develop at least one PU in their lifetime. ${ }^{11} \mathrm{PUs}$ have a negative impact on quality of life, ${ }^{12}$ contribute towards rehabilitation set-backs and hospitalisation, ${ }^{13}$ in $7-8 \%{ }^{14}$ patients complications that can lead to death, ${ }^{9}$ and significantly impact on the life-long care management of SCI patients.

The 2009 NPUAP/EPUAP evidence-based risk assessment for $\mathrm{PU}$ prevention and management statement ${ }^{1}$ was developed to guide clinicians towards the most appropriate course of action for preventing and managing PUs in health-care settings. The guidelines were formulated based on a comprehensive generic literature and although the collaboration considered the unique needs of SCI patients, relevant SCI-specific literature was not reviewed comprehensively. Consequently, the specific needs of this patient population were not adequately represented.

Recently, risk factors (RFs) for PU development in the general adult population have been reviewed. ${ }^{15}$ No single factor can explain PU risk; rather, there is a complex interplay of factors that increase the probability of PU development. Three independent predictors of PU development emerged: mobility, perfusion (including diabetes) and skin condition. Other important factors include skin moisture, age, haematological measures, nutrition and general health status. ${ }^{16-18}$ Gender, marital status, ${ }^{19,20}$ education, income and time from injury $^{21}$ have been associated with the presence of PUs in people with SCI, suggesting these factors may be important predictors of PU development in SCI. Longitudinal research may assist in determining RFs independently predictive of PU development. Considering that 
people with SCI may be both insensate and immobile, ${ }^{16}$ elucidation of key RFs for PUs would enable identification of those people with SCI who are most susceptible to PUs and subsequently implementation of appropriate preventative interventions. This would require differentiating key RFs for people with SCI from those of the general population to accurately predict $\mathrm{PU}$ development in SCI and to guide the development of precise and specific risk assessment scales and clinical care guidelines for SCI patient care.

This research sought to identify RFs that independently predict PU development and recurrence in adult SCI populations and determine whether RFs specific for people with SCI differ from RFs for the general PU population. ${ }^{15}$

\section{MATERIALS AND METHODS}

\section{Research design}

A systematic review including prospective cohort, retrospective record reviews and clinical trials that identified RFs associated with PU development and recurrence in SCI populations using multivariate analytical techniques.

\section{Electronic search}

MEDLINE and EMbase databases were searched from January 1980 to September 2011 to identify relevant studies electronically. A search strategy was developed consisting of search terms for PUs (for example, pressure sore, bed sore or decubitus ulcer) and SCI (for example, spinal injury, paralysis or quadraplegic). ${ }^{22}$ To increase specificity, the searches were refined by publication (not commentary, letter, guidelines or audit), wound type (not buruli, digital ulcers, burns or spider bites), and limited to humans and adults (aged 18 years or above). The search strategy is available on request. The Cochrane Clinical Trials Register was also searched using terms: 'pressure ulcer or pressure sore' and 'spinal cord or spinal injury' and trial. Searches were restricted to adult, human studies. No language or geographical restrictions were applied. Authors of non-English papers were contacted to provide information required to determine eligibility. A citation search was performed on studies meeting eligibility criteria to identify further studies not retrieved electronically.

\section{Study selection criteria}

Eligibility criteria stage 1. Retrieved abstracts were reviewed against the eligibility criteria by two reviewers independently (JM, CG). Prospective cohort or retrospective record review studies, with or without comparison groups, or controlled trials of adult in- or outpatients, in acute, intermediate or long-term care settings with a SCI were considered potentially relevant if the outcome was the development of a new PU of any category or location and/or recurrence of a previous PU. No restrictions were applied by type of RFs studied.

Eligibility criteria stage 2. Studies were included if: the study inclusion criteria were clearly reported; outcome was either development of PU $\geqslant$ category 1 or equivalent or recurrence; and analyses included multivariate methods to identify RFs associated with PU development. In addition, cohort and record review studies were included if loss to follow up was less than $20 \%$; clinical trials were included if allocation concealment and intention to treat methods were used. Potentially relevant studies identified at stage 1 were obtained and assessed by two reviewers (JM, CG) independently against stage two criteria. Disagreements were discussed with a third reviewer (JN).

\section{Quality criteria/assessment}

Quality criteria, consistent with methods developed by Nixon et al. ${ }^{15}$ and based upon guidelines for assessing quality in prognostic studies and methodological considerations in the analysis and publication of observational studies, ${ }^{23,24}$ were applied to studies meeting stage two criteria. Each study was appraised by two reviewers (JN, CG) and the following methodological limitations were noted where present: baseline characteristics not adequately described; inadequate measurement of RFs; inappropriate cut-points used for continuous data; and time-dependent covariate's included in the analysis without appropriate adjustment.

In addition, specific consideration was given to the following criteria:

1. Was there sufficient number of events (rule of thumb, $\geqslant 10$ events per RF)

2. Was there sufficient presentation of data to assess the adequacy of method and analysis

3. Is the strategy for model building (that is, inclusion of variables) appropriate

4. Was the selected model adequate for the design

Each criterion was assessed as being met (yes/no/partial/unsure), which provided a structured approach for the classification of overall study quality:

- High: yes to all criteria

- Medium: yes to criteria 1 and at least 2 other criteria

- Low: no for criteria 1 and not or partial for 2 other criteria

- Very low: no for criteria 1 and not or partial for all 3 other criteria

\section{Data extraction}

For studies that met stage 2 eligibility criteria, data were extracted by a single reviewer (JM) into tables and checked by a second reviewer (CG). Study characteristics (for example, study population, recruitment type used, PU definition and analysis method) and RFs investigated in the multivariate models, including those found to be significant, were extracted. Authors were contacted if papers were unobtainable or methods reported needing clarification.

\section{Data synthesis}

Study heterogeneity did not allow for meta-analysis to be conducted. As the aim of this review was to identify RFs and not quantify their effects on PU development, a narrative synthesis was conducted to present RFs. Significant RFs were extracted, their frequency reported and summarised under relevant themes. The provisional RF themes that emerged were directly compared with PU RFs identified for the general population. ${ }^{15}$

Study RFs entered into multivariate modeling, as well as those which emerged as significant $(P=\leqslant 0.05)$ in each study, were recorded. Those using stepwise regression, RFs were recorded $(P=\geqslant 0.05)$ if they were independently associated with PU development in the final model. Significant RFs were then categorised to one of six themes and their significance levels recorded.

\section{RESULTS}

The searches retrieved 549 citations, minus duplicates. Of these, 46 were considered potentially eligible, and 5 studies met the eligibility criteria (Figure 1). The five included studies represent 595 participants with PUs (sample sizes ranged from 64 to 193) from acute, community and long-term care settings across the United States and Holland (Table 1). Two studies were classified as high quality ${ }^{25,26}$ and three studies as low quality ${ }^{27-29}$ (Table 2).

Combined, the five studies explored 23 RFs through multivariate analyses, of which 18 emerged as significant and were grouped into six themes: socio demographic, neurological, functional, clinical biological and medical care management (Table 3). Completeness of injury, level of lesion, functional independence measure (FIM) level of self-care and independence, pulmonary condition, ${ }^{25,26,28}$ CHART level of independence, previous PU surgery and self-reports ${ }^{30}$ were significant RFs for PUs in people with SCI. For PU recurrence, ${ }^{27}$ ethnicity, comorbidity and sitting time at discharge were important RFs (Table 3).

\section{DISCUSSION}

This systematic review has identified RFs associated with the development and recurrence of PUs in the SCI population. Important RFs include SCI lesion characteristics, motor independence/mobility 


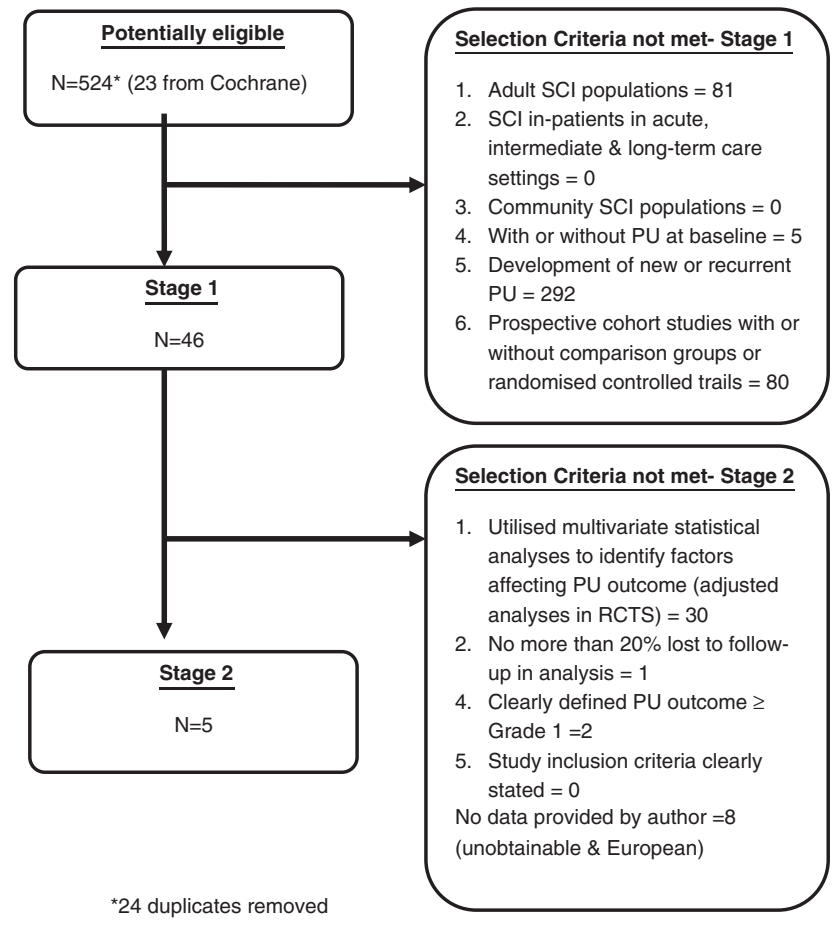

Figure 1 Flow diagram of studies retrieved and included.

and having a history of PUs, compared mobility, perfusion and skin condition for the general PU population. ${ }^{15}$ Additional predictors of PU development and recurrence in SCI include clinical, functional and hospital management domains. Mobility was found to be a significant RF for both SCI and general PU populations. ${ }^{15}$ For SCI patients, a range of assessment scales were used, both specifically (for example, American Spinal Injury Association Impairment Scale ${ }^{30}$ ) and not specifically designed for people with SCI (for example, Functional Independence Measure ${ }^{31}$ ). This lack of homogeneity in descriptor values impacts upon the interpretation and use of the data in further analysis, suggesting a need for an agreed minimum data set that includes mobility and other SCI RF variables such as SCI lesion characteristics.

Initial medical care management was a significant RF for PU development in people with SCI, findings consistent with Gélis et al. ${ }^{32}$ Quick admission to a specialist unit and the use of the correct pressure-relieving equipment can reduce $\mathrm{PU}$ incidence. ${ }^{33}$ The development of a PU increases patients' risk of further PU development and recurrence, ${ }^{34}$ leading to added medical complications. African American origin was also found to be a RF for PU development in SCI; it may be more difficult to recognise skin breakdown in individuals with darker skin..$^{31,35,36}$ However, race may be a proxy for social factors such as wealth, education and health-care accessibility. ${ }^{31,36}$ Further research is needed to determine the extent of the relationship between socioeconomic, racial and clinical factors, and how they affect PU development. A coordinated multidisciplinary team response to a SCI patient may be required to reduce length of hospital stay, improve quality of life and reduce health-care costs; however, further empiric evidence is required.

There are numerous RFs related to tissue perfusion making it a multi-factorial risk. 'Perfusion-related variables' such as diabetes, anaemia and blood pressure are thought to increase PU development. ${ }^{16}$ Although excluded in this review due to recall bias, patient self-report prevalence surveys have found a link between tobacco use and PU development in people with SCI. ${ }^{37,38}$ Age has been a disputed RF for both the general ${ }^{39,40}$ and the SCI population. ${ }^{38}$ Age is likely to be a multi-factorial RF related to increased morbidity and disease, which decreases mobility and in turn affects skin tolerance and its ability to heal. ${ }^{17}$ There may be an association between age, skin perfusion and ability of skin to heal, but no definitive study has found a link. Perfusion is a broad theme and further investigation is needed to determine how the array of variables interact and affect skin breakdown, and consequently PU risk in SCI patients.

$\mathrm{RF}$ research in SCI has focused on factors associated with the injury itself, such as the level and completeness of injury and when and how it occurred. ${ }^{25,32,36}$ In contrast, RF research in the general PU population has often focused on factors such as moisture, perfusion and nutrition. ${ }^{15}$ It is therefore unclear whether our findings are due to a research bias or whether in fact the two populations have different PU RFs (for example, not all RFs associated with PU development in the general population have been investigated as potential RFs for people with SCI).

Our findings show no definitive difference between RFs for PU development and recurrence; co-morbidity and ethnicity were highlighted for both, however, time spent sitting at hospital discharge was only associated with recurrence. However, only one study investigated RFs associated with PU recurrence, highlighting the need for further research. Some might suggest that RFs associated with development of new PUs are different to RFs associated with recurrence but this was outside the scope of this review and requires formal review and further analysis to inform future research and clinical practice. In the general and SCI populations, having a history of PUs was a RF for future PU development. ${ }^{34}$ Decreased mobility as a result of surgery or an existing PU increased the risk of further PU development/ recurrence, particularly on bony prominences. ${ }^{31,36}$ PUs can also cause changes in the skin, increasing susceptibility to PU recurrence/development. ${ }^{41}$ Current risk assessment does not contain $\mathrm{PU}$ history as a $\mathrm{RF}^{36}$ despite mounting evidence that it is an important RF. SCI-specific risk assessment scales may need revising in light of the review finding.

Our work builds on previous research ${ }^{15,32,36,42}$ through adapted methods, a comprehensive search of the literature, and strict eligibility criteria applied, including only studies that had used multivariate statistical methods. We also undertook a thorough quality assessment, with consideration of study attrition, participation and confounding factors as a means of highlighting study bias and limitations. Two of the studies included in this review used rigorous analytical techniques to identify significant RFs. However, three were assessed as low quality, mainly due to insufficient number of events recorded per RF and therefore not powered appropriately for multivariate analyses. This has implications for other findings; it is possible that not all RFs important in PU development or recurrence in people with SCI were identified as significant. Evidence suggests factors such as age at injury, duration of injury and the presence of an indwelling catheter may be important RFs, but these did not emerge as significant in models. Thus, these should not be disregarded as potential RFs associated with PU development or recurrence until further investigation precludes them.

Our review highlights important limitations with the current evidence and methodological challenges associated with interpretation of RF reviews in the absence of clear guidelines. Few studies have explored RFs for PUs in people with SCI. The few that have explored RFs in multivariate analyses explored different factors across studies, 


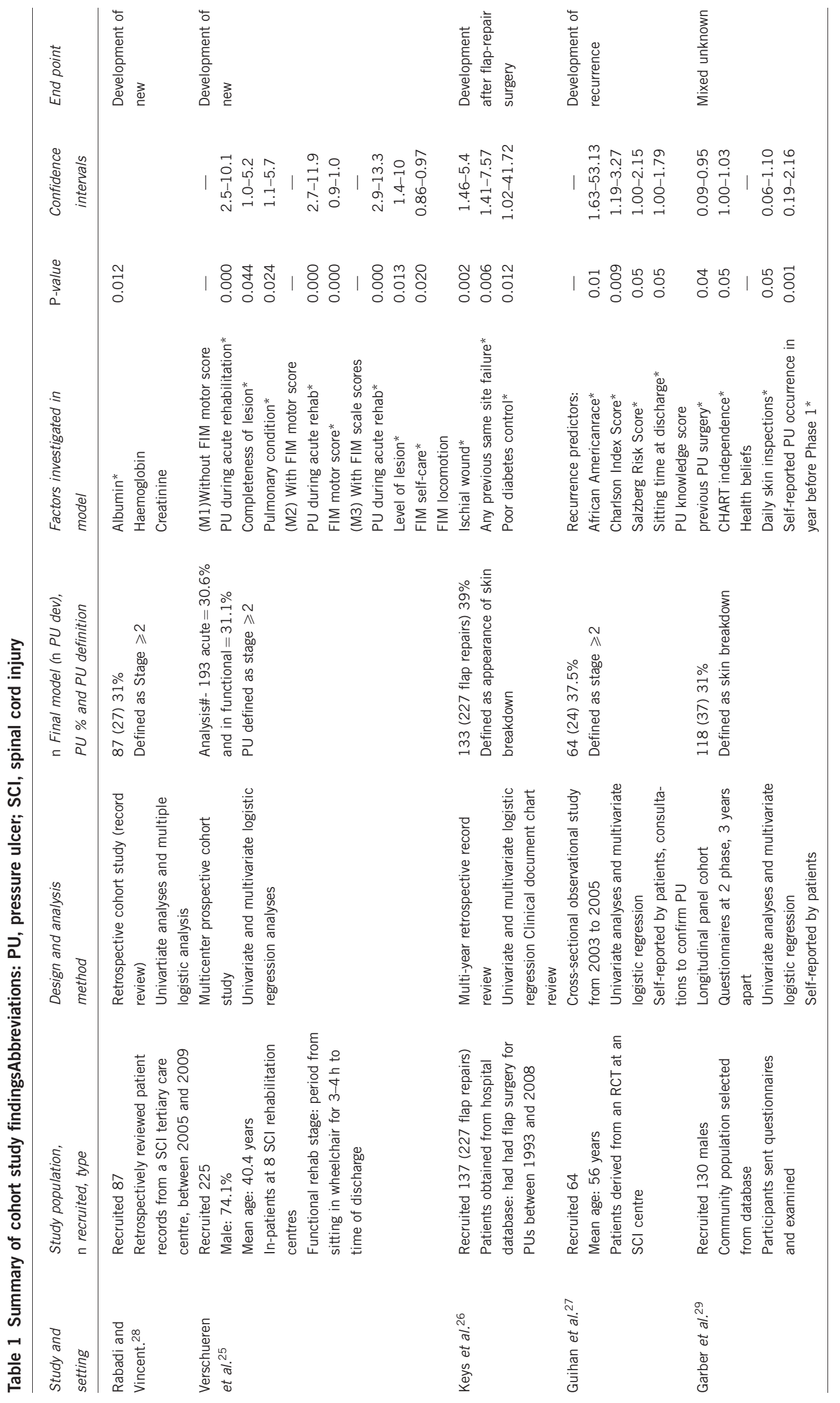


Table 2 Summary of quality of included studies

\begin{tabular}{lcl}
\hline Quality of study & $\begin{array}{c}\text { Number of } \\
\text { studies }\end{array}$ & $\begin{array}{l}\text { Specific studies } \\
\text { (by first author name) }\end{array}$ \\
\hline High & 2 & Verschueren, ${ }^{25}$ Keys $^{26}$ \\
Medium & 0 & \\
Low & 3 & Rabadi, $^{28}$ Guihan, $^{27}$ Garber $^{29}$ \\
Very low & 0 & \\
\hline
\end{tabular}

Table 3 Summary of significant RFs

\begin{tabular}{|c|c|c|c|c|c|c|}
\hline Domain & $R F S$ & Rabadi $^{28}$ & Verscheuren ${ }^{25}$ & Garber $^{29}$ & Keys ${ }^{26}$ & Guihan $^{27}$ \\
\hline Sociodemographic & Ethnicity & & & & & 0.010 \\
\hline \multirow{2}{*}{ Neurological } & Completeness & & 0.044 & & & \\
\hline & $\begin{array}{l}\text { Level of } \\
\text { lesion }\end{array}$ & & 0.013 & & & \\
\hline \multirow[t]{3}{*}{ Functional } & CHART & & & 0.050 & & \\
\hline & $\begin{array}{l}\text { Independence } \\
\text { FIM self-care }\end{array}$ & & 0.020 & & & \\
\hline & $\begin{array}{l}\text { FIM motor } \\
\text { independence }\end{array}$ & & 0.000 & & & \\
\hline \multirow[t]{6}{*}{ Clinical } & Comorbidity & & & & & 0.009 \\
\hline & $\begin{array}{l}\text { Previous PU } \\
\text { surgery }\end{array}$ & & & 0.040 & & \\
\hline & $\begin{array}{l}\text { Same site } \\
\text { failure }\end{array}$ & & & & 0.006 & \\
\hline & Ischial wound & & & & 0.002 & \\
\hline & $\begin{array}{l}\text { PU during } \\
\text { rehabilitation }\end{array}$ & & 0.000 & & & \\
\hline & $\begin{array}{l}\text { Pulmonary } \\
\text { condition }\end{array}$ & & 0.024 & & & \\
\hline \multirow[t]{2}{*}{ Biological } & Albumin & 0.020 & & & & \\
\hline & $\begin{array}{l}\text { Poor diabetes } \\
\text { control }\end{array}$ & & & & 0.012 & \\
\hline \multirow[t]{5}{*}{$\begin{array}{l}\text { Medical care } \\
\text { management }\end{array}$} & $\begin{array}{l}\text { Self-report of } \\
\text { PU }\end{array}$ & & & & & \\
\hline & $\begin{array}{l}\text { Daily skin } \\
\text { inspection }\end{array}$ & & & 0.050 & & \\
\hline & Salzberg Risk & & & & & 0.050 \\
\hline & $\begin{array}{l}\text { Score } \\
\text { Sitting time }\end{array}$ & & & & & 0.050 \\
\hline & at discharge & & & & & \\
\hline
\end{tabular}

Abbreviations: PU, pressure ulcer; RF, risk factor.

astudy investigating the recurrence of PUs.

limiting interpretation and overall conclusions. Further, the heterogeneity and methodological limitations of the studies identified precluded combining study results using meta-analysis. The number of studies included in this review was small, partly due to the strict inclusion criteria but also because of the lack of research conducted in this area. Due to the nature of their design, RFs highlighted in prevalence studies were considered prognostic indicators, giving frequency and distribution of SCI RFs. Including prognostic data assisted in the identification of factors that might be important for PU development, such as psychological factors ${ }^{38,43}$ and drug and alcohol use; 35 factors not explored by the studies included in this review. However, subsequent cohort studies are needed to determine the significance of these factors. Prevalence studies provide descriptive evidence for RFs and enable the tracking of trends, comparison of populations and time-point information, ${ }^{44}$ but do not provide information pertaining to cause and effect. Including cohort studies using multivariate analysis techniques to identify RFs provided insight into the relationships between variables ${ }^{45}$ through greater statistical power; however, it does not preclude other factors as potentially important for PU development; those identified through univariate or other methods.

Failing to undertake a meta-analysis may be perceived as a limitation, however, a question arises as to whether it is possible. Simon and Altman ${ }^{46}$ suggest that meta-analysis is far more problematic for combining prognostic factor studies compared with clinical trials. The main problem is that patient inclusion criteria is not standardised across studies and prognostic studies often give no quantitative information beyond a $P$-value (for example, only 'not significant' reported). Negative findings are seldom published, suggesting that there may be more publication bias than has previously been reported for clinical trials. ${ }^{47}$ Meta-analysis could not be effectively performed using only the information available from published research in this field. Therefore, a narrative synthesis of RFs that independently predicted the development and recurrence of PUs in the SCI population was undertaken, focussing on studies that had used rigorous statistical methods.

\section{CONCLUSION}

Work to identify RFs that independently predict PUs has been undertaken, however, factors specific to the SCI population have not been fully determined. This systematic review identified SCI-specific RFs for the development and recurrence of PUs. Skin quality and mobility are important RFs for both populations; however, characteristics of the spinal lesion, existing/previous PUs and independence in PU management are important SCI-specific RFs. Research in this area is scarce, highlighting the need for further confirmatory work to reduce PU development and recurrence and provide a foundation for SCI risk assessment development. Owing to differences identified between PU RFs for the general and SCI populations, different approaches to the care and management of these patients may be needed.

\section{DATA ARCHIVING}

There were no data to deposit.

\section{CONFLICT OF INTEREST}

The authors declare no conflict of interest.

1 National Pressure Ulcer Advisory Panel and the European Pressure Ulcer Advisory Panel (NPUAP/EPUAP). Prevention and Treatment of Pressure Ulcers: Clinical Practice Guideline. NPUAP: Washington DC, 2009, p 169.

2 Witkowski JA, Parish LC. Histopathology of the decubitus ulcer. J Am Acad Dermatol 1982; 6: 1014-1021.

3 Vanderwee K, Clark M, Dealey C, Gunningberg L, Defloor T. Pressure ulcer prevalence in Europe: a pilot study. J Eval Clin Pract 2007; 13: 227-235.

4 Whittington K, Patrick M, Roberts JL. A national study of pressure ulcer prevalence and incidence in acute care hospitals. J Wound Ostomy Cont 2000; 27: 209-215.

5 James J, Evans J, Young T, Clark M. Pressure ulcer prevalence across Welsh orthopaedic units and community hospitals: surveys based on the European Pressure Ulcer Advisory Panel minimum data set. Int Wound J 2010; 7: 147-152.

6 Kaltenthaler E, Whitfield MD, Walters SJ, Akehurst RL, Paisley S. UK, USA and Canada: How do their pressure ulcer prevalence and incidence data compare? J Wound Care 2001; 10: 530-535.

7 Bennett G, Dealey C, Posnett J. The cost of pressure ulcers in the UK. Age Ageing 2004; 33: 230-235.

8 Rycroft-Malone J. Pressure ulcer risk assessment and prevention: clinical practice guidelines. Royal College of Nursing: London, 2001.

9 McKinley WO, Jackson AB, Cardenas DD, DeVivo MJ. Long-term medical complications after traumatic spinal cord injury: a regional model systems analysis. Arch Phys Med Rehabil 1999; 80: 1402-1410.

10 Haisma JA, Van der Woude LH, Stam HJ, Bergen MP, Sluis TA, Post MW. Complications following spinal cord injury: occurrence and risk factors in a longitudinal study during and after inpatient rehabilitation. J Rehabil Med 2007; 39: 393-398.

11 Gunnewicht BR. Pressure sores in patients with acute spinal cord injury. $J$ Wound Care 1995; 4: 452-454.

12 Gorecki C, Brown JM, Nelson EA, Briggs M, Schoonhoven L, Dealy C et al. Impact of pressure ulcers on quality of life in older patients: a systematic review. J Am Geriatr Soc 2011; 57: 1175-1183.

13 Cardenas DD, Hoffman JM, Kirshblum S, McKinley W. Etiology and incidence of rehospitalization after traumatic spinal cord injury: a multicenter analysis. Arch Phys Med Rehabil 2004; 85: 1757-1763. 
14 Byrne DW, Salzberg CA. Major risk factors for pressure ulcers in the spinal cord disabled: a literature review. Spinal Cord 1996; 34: 255-263.

15 Coleman S, Gorecki C, Nelson EA, Closs J, Defloor T, Halfens R et al. Patient risk factors for pressure ulcer development: systematic review. Int J Nurs Stud 2013, (doi:org/10.1016/j.ijnurstu.2012.11.019).

16 Nixon J, McGough A. Principles of patient assessment: Screening for pressure ulcers and potential risk. In: Morison MJ (ed). The Prevention and Treatment of Pressure Ulcers, 1st edn. Harcourt: London, 2001, pp 55-74.

17 Allman RM. Pressure ulcer prevalence, incidence, risk factors and impact. Clin Geriatr Med 1997; 13: 421-436.

18 Brandeis GH, Ooi WL, Hossain M, Morris JN, Lipsitz LA. A longitudinal study of risk factors associated with the formation of pressure ulcers in nursing homes. J Am Geriatr Soc 1994; 42: 388-393.

19 Chen Y, DeVivo MJ, Jackson AB. Pressure ulcer prevalence in people with spinal cord injury: Age-period-duration effects. Arch Phys Med Rehabil 2005; 86: 1208-1213.

20 Taghipoor KD, Arejan RH, Rasouli MR, Saadat S, Moghadam M, Vaccaro AR et al. Factors associated with pressure ulcers in patients with complete or sensory-only preserved spinal cord injury: Is there any difference between traumatic and nontraumatic causes? J Neurosurg Spine 2009; 11: 438-444.

21 Saunders LL, Krause JS, Peters B, Reed KS. Relationship of pressure ulcers, race and socioeconomic status after SCl. J Spinal Cord Med 2010; 33: 387-395.

22 The Cochrane Collaboration, 2009. The Cochrane Library. Retrieved from http:// www.thecochranelibrary.com/view/O/index.html.

23 Hayden JA, Côté $P$, Bombardier C. Evaluation of the quality of prognosis studies in systematic reviews. Ann Intern Med 2006; 144: 427-437.

24 Von Elm E, Altman G, Egger M, Pocock SJ, Gotzsche C, Vandenbroucke JP. The strengthening the reporting of observational studies in epidemiology (STROBE) statement: guidelines for reporting observational studies. B World Health Organ 2007; 85: 867-872.

25 Verschueren JHM, Post MWM, de Groot S, Van Der Woude LHV, Van Asbeck FWA, Rol M. Occurrence and predictors of pressure ulcers during primary in-patient spinal cord injury rehabilitation. Spinal Cord 2011; 49: 106-112.

26 Keys KA, Daniali LN, Warner KJ, Mathes DW. Multivariate predictors of failure after flap coverage of pressure ulcers. Plast Reconstr Surg 2010; 125: 1725-1734.

27 Guihan M, Garber SL, Bombardier CH, Goldstein B, Holmes SA, Cao L. Predictors of pressure ulcer recurrence in veterans with spinal cord injury. J Spinal Cord Med 2008; 3: 551-559.

28 Rabadi MH, Vincent AS. Do vascular risk factors contribute to the prevalence of pressure ulcers in veterans with spinal cord injuries. J Spinal Cord Med 2011; 34: 46-51.

29 Garber SL, Rintala DH, Hart KA, Fuhrer MJ. Pressure ulcer risk in spinal cord injury: Predictors of ulcer status over 3 years. Arch Phys Med Rehabil 2000; 81: 465-471.

30 Waters RL, Adkins R, Yakura J, Vigil D. Prediction of ambulatory performance based on motor scores derived from standards of the American Spinal Association. Arch Phys Med Rehabil 1994; 75: 756-760.
31 Keith RA, Granger CV, Sherwin FS. The functional independence measure: a new tool for rehabilitation. Adv Clin Rehabil 1987; 1: 6-18.

32 Gélis A, Dupeyron A, Legros P, Benaim C, Pelissier J, Fattal C. Pressure ulcer risk factors in persons with SCI: Part I: acute and rehabilitation stages. Spinal Cord 2009; 47: 99-107.

33 Celani MG, Spizzichino L, Ricci S, Zampolini M, Franceschini M. Retrospective Study Group on SCl. Spinal cord injury in Italy: a multicenter retrospective study. Arch Phys Med Rehabil 2001; 82: 589-596.

34 Niazi ZB, Salzberg CA, Byrne DW, Viehbeck M. Recurrence of initial pressure ulcer in persons with spinal cord injuries. Adv Wound Care 1997; 10: 38-42.

35 Waters RL, Adkins RH, Sie I, Cressy J. Postrehabilitation outcomes after spinal cord injury caused by firearms and motor vehicle crash among ethnically diverse groups. Arch PhysMed Rehabil 1998; 79: 1237-1243.

36 Gélis A, Dupeyron A, Legros P, Benam C, Pelissier J, Fattal C. Pressure ulcer risk factors in persons with spinal cord injury Part 2: the chronic stage. Spinal Cord 2009; 47: 651-661.

37 Krause JS, Vines CL, Farley TL, Sniezek J, Coker J. An exploratory study of pressure ulcers after spinal cord injury: relationship to protective behaviors and risk factors. Arch Phys Med Rehabil 2001; 82: 107-113.

38 Krause JS, Broderick L. Patterns of recurrent pressure ulcers after spinal cord injury: identification of risk and protective factors 5 or more years after onset. Arch Phys Med Rehabil 2004; 85: 1257-1264.

39 Slowikowski GC, Funk M. Factors associated with pressure ulcers in patients in a surgical intensive care unit. J Wound Ostomy Cont 2010; 37: 619-626.

40 Lardenoye JW, Thiefaine JA, Breslau PJ. Assessment of incidence, cause, and consequences of pressure ulcers to evaluate quality of provided care. Dermatol Surg 2009; 35: 1797-1803.

41 Frontera WR, Silver JK, Rizzo TD. Essentials of Physical Medicine and RehabilitationMusculoskeletal Disorders, Pain and Rehabilitation, 2nd edn. Saunders Elsevier: Philadelphia, 2008.

42 Gorecki C, Closs SJ, Nixon J, Briggs M. Patient-reported pressure ulcer pain: a mixedmethods systematic review. J Pain Symptom Manage 2010; 42: 443-459.

43 New PW, Rawicki HB, Bailey MJ. Nontraumatic spinal cord injury rehabilitation: pressure ulcer patterns, prediction, and impact. Arch Phys Med 2001; 85: 87-93.

44 Radulescu M, Diepgen T, Williams $\mathrm{H}$. What makes a good prevalence survey? In: Williams H, Bigby M, Diepgen T, Herxheimer A, Maldi L and Rzany B (eds). Evidence Based Dermatology, 2nd edn. Blackwell: Oxford, 2008, pp 61-67.

45 Khattree R, Naik DN. Applied Multivariate Statistics: With SAS Software, 2nd edn. Cary, 1999.

46 Simon R, Altman DG. Statistical aspects of prognostic factor studies in oncology. Br J Cancer 1994; 69: 979-985.

47 Sterne JAC, Egger M, Smith GD. Systematic reviews in health care: investigating and dealing with publication and other biases in meta-analysis. BMJ 2001; 323: 101-105. 